\title{
Potential therapeutic effects of phytochemicals and medicinal herbs for cancer prevention and treatment.
}

\author{
Elyn C Jacobs* \\ Former Executive Director, Emerald Heart Cancer Foundation, USA
}

\begin{abstract}
Cancer has a major impact on society across the world. It is one of the major causes of death, with approximately 1.7 million new cases expected to be diagnosed in the United States and 600,000 people will die from the disease. Worldwide statistics in 2012 reported over 14 million new cases and 8.2 million cancer-related deaths. New cases are expected to rise by about $\mathbf{7 0 \%}$ over the next 20 years. The current standard of care, surgery, chemotherapy, and radiation has not proved to be highly effective and comes with significant side effects and costs. Although conventional cancer therapy can target certain cancer cells and sometimes prevent relapse of the illness, complementary and alternative therapies are required due to disadvantages of the current therapies such as low effectiveness, adverse effects, or availability. Moreover, a number of studies have found adjunctive therapeutic approaches to improve outcomes for cancer patients. One potential complementary method with conventional cancer drugs involves the use of medicinal herbs and phytochemicals. The possible therapeutic benefits include, but are not limited to antiproliferative, apoptotic, anti-metastatic, and anti-angiogenic effects, of which have been demonstrated in in vitro experiments and some clinical trials; more clinical trials need to be done. This review summarizes some plant sources exhibiting anti-cancer and chemoprevention activity. An overview of how phytochemicals and medicinal herbs permit the prevention and ultimate removal of cancer cells with minimal trauma to the host will be presented.
\end{abstract}

Keywords: Phytochemicals, Medicinal herbs, Chemoprevention.

\section{Introduction}

Cancer is still one of the major causes of mortality in both developing and developed countries. At present, in spite of intensive interventions, a large number of patients suffer from poor prognosis. Therefore, there has been an effort towards finding new anticancer agents with better efficacy and fewer side effects. Numerous medicinal plants have been reported to have chemopreventive and therapeutic effects. Antiproliferative, pro-apoptotic, anti-metastatic, and anti-angiogenic effects of phytochemicals and medicinal herbs have been shown in in vitro experiments or animal studies and many have been documented in clinical trials. Studies have revealed beneficial effects of medicinal herbs and phytochemicals against cancer by providing anti-inflammatory, anti-bacterial, antiviral, free radical scavengers in the form of cancer-fighting compounds. Phytomedicines have the ability to interact with signaling pathways that modulate cell growth, replication, and death of a wide variety of tumor cell types through diverse mechanisms. These phytocomplexes have the remarkable capability of affecting us on a cellular level-where diseases such as cancer originate. Many plant species are already being used to treat or prevent development of cancer outside of the traditional oncology environment, notably without harm to healthy cells.

The influence of natural products upon drug discovery in general has been quite impressive; one only has to look at the number of clinically active drugs that are in use in cancer therapy to see how many either are natural products or have a natural pro-duct pharmacophore. Over the time frame of the
1940 s to the end of $2014,49 \%$ of cancer drugs were derived from natural compounds [1]. One example is the popular chemo drug Taxol, which is made from the bark of the Pacific Yew tree (Taxus). Unfortunately, it appears to be difficult to engineer a chemically-derived drug which is non-toxic to normal cells and is specific to cytotoxicity of cancer cells. Yet, despite numerous accounts of their powerful effects on cancer in their natural states these phytomedicines have been largely ignored for the prevention and treatment of cancer. There are two sections in this review, phytochemicals against cancer and medical herbs against cancer.

\section{Phytochemicals and Cancer}

Phytochemicals are biologically active compounds found in plants which have protective and disease-preventive properties. They have shown to exhibit chemoprevention and chemotherapeutic effects not only in cell lines but also in some clinical trials. This section offers a few examples of plantderived compounds that have anticancer therapeutic properties and which offer no harm to healthy cells.

\section{Quercetin}

Found in grapes, onions, apples, red wine, licorice root, and berries, quercetin is a powerful anticancer, anti-inflammatory, and antiviral agent. It has been found to inhibit various types of cancer such as breast, liver, leukemia, colon, ovary, endometrial, gastric and non-small-cell lung cancers. Quercetin has anti-estrogenic effects and therefore may reduce the metastatic potential of breast cancer cells. Research shows that 
quercetin controls cancer cell growth through the regulation of signaling pathways such as activating tumor suppressor genes (such as p53 and p21), inducing apoptosis (cancer cell death), and inhibiting angiogenesis (the ability of cells to make new blood vessels). Several studies have found that quercetin can significantly inhibit liver cancer (hepatic cancer) cell proliferation through the regulation of cyclin D1 expression. Researchers have reported delayed tumor growth, increased tumor necrosis, and improved survival rates [2]. Other research shows that quercetin inhibits tyrosine kinase, one of the major enzymes associated with cancer and helps flush cancerpromoting chemicals such as BPA (Bisphenol-A) from the body. Importantly, quercetin could be an effective antiviral and antitumor agent against Epstein-Barr Virus (EBV) infection and the human carcinomas it is involved with [3]. Quercetin induces signal transductions to stimulate apoptosis and induces EBV gene transcription; quercetin-induced apoptosis appears to prevent EBV infection.

\section{Resveratrol}

Found in red wine, red grapes, and dark berries, resveratrol inhibits cancer by reducing cell proliferation and metastasis and by inducing apoptosis [4]. In particular, resveratrol acts against several genes that promote prostate, colorectal, and other cancers [5]. It inhibits COX-2 expression which in turn inhibits angiogenesis [6]. Resveratrol also activates the tumor suppressor gene $\mathrm{p} 53$, which regulates cell division by keeping cells from growing and dividing too fast or in an uncontrolled way. P53 plays a critical role in determining whether damaged DNA will be repaired or a damaged cell will self-destruct (undergo apoptosis). If the DNA can be repaired, p53 activates other genes to fix the damage. If the DNA cannot be repaired, this protein prevents the cell from dividing and signals it to undergo apoptosis. By stopping cells with mutated or damaged DNA from dividing, p53 helps prevent the development of tumors. Resveratrol has also been found to alter the expression of other genes involved in cell cycle regulation and apoptosis, including cyclins, cdks, and cdk inhibitors [7]. It also inhibits the gene that makes aromatase, the enzyme that controls estrogen production, inhibiting the growth of estrogen-sensitive cancers [8].

\section{Mistletoe constituents}

Mistletoe is one of the most widely studied alternative therapies for cancer. It has been used as an overall treatment for cancer for years. One of the most commonly used oncological drugs in Europe is iscador, which is derived from extracts of mistletoe.

One randomized and nonrandomized matched pair study conducted in Germany that involved more than 10,000 cancer patients found iscador to prolong the survival time of cancer patients by as much as $40 \%$ [9]. Numerous other studies that show the positive effects of this plant on cancer patients. A Phase I clinical trial for mistletoe is currently being conducted at Johns Hopkins University School of Medicine, but it will likely take many years for the FDA to approve it for cancer treatment in the United States.

\section{Lycopene}

Lycopene is a phytochemical found in abundance in watermelon and tomato products (particularly when cooked). Studies show efficacy towards cancers of the pancreas, colon, rectum, esophagus, oral cavity, stomach, lungs, prostate, breast, and cervix [10]. For example, a 2016 review published by the American Institute for Cancer Research suggested that eating tomatoes and other lycopene-containing foods lowers prostate cancer risk [11]. A 2017 review showed lycopene has specific effects on the proliferation and progression of colon cancer. It is believed that the beneficial effects may be in part of lycopene's ability to interact with signaling pathways that modulate cell growth, replication, and death [12]. One of those pathways is insulin-like growth factor-1 (IGF-1). A human study found that $30 \mathrm{mg}$ of lycopene daily reduced IGF-1 by $25 \%$ [13].

\section{Selenium}

Selenium has long been thought of as a potent anti-cancer compound. It has the ability to alter the metabolism of some carcinogens and can be effective in inhibiting the initiation phase of cancer. A diet rich in selenium protects against cancers such as those of the stomach, breast, esophagus, lung, tongue, liver, colon, kidneys, bladder, pancreas, prostate, colon, and rectum. In a 1996 study conducted by Dr. Larry Clarke at the University of Arizona and published in the Journal of the American Medical Association, increased selenium lowered the incidence of cancers of the lung, colon, and prostate by roughly $50 \%$ [14]. The researchers found that the higher the level of selenium, the lower the incidence of tumor formation, bringing the risk-reduction even higher for some cancers.

Selenium enhances the effectiveness of chemo and radiation therapies while reducing the toxicity to normal cells. For example, selenium helps reduce the risk of heart damage from Adriamycin and toxicity to the kidneys and bone marrow suppression induced by Cisplatin. Selenium is necessary for liver detoxification, a process essential for the metabolism of estrogen and other toxins such as heavy metals, chlorine, fluoride, and bromide. It supports immune function, helps prevent tumors from developing, and contributes to the death of pre-cancerous and cancerous cells. It inhibits virally-induced cancers and helps repair DNA damage from carcinogens. Selenium is abundant in brazil nuts. It can also be found in cruciferous vegetables such as broccoli, kale, and cauliflower. Shitake mushrooms, white button mushrooms, lima beans, pinto beans, and seeds such as chia, sunflower, sesame, and flax also contain selenium.

\section{Medicinal Herbs and Cancer}

Of the thousands of herbs that grow around the world, Western herbalists consider around 200 to be of primary therapeutic value (traditional Chinese medicine recognizes over 400). Science is increasingly backing up what herbalists have known for centuries making herbal medicine available for the treatment of disease through Complementary and Alternative Medicine programs (CAM), Integrative Medicine (IM), naturopathic medicine, and often self-care. Below are a few notable herbs. In most cases human clinical trials examining the effects of medicinal herbs for cancer prevention and treatment have not been conducted although there is considerable potential for herbs to be utilized as chemo preventive agents.

\section{Turmeric}

One of the most powerful and protective herbs and the one 
that continues to show the most promise when it comes to fighting and preventing cancer, is turmeric. It is also the most widely studied herb. Part of the ginger family, turmeric is a major component used in both Chinese and Indian medicine. It derives its power from the compound curcumin, which is responsible for giving turmeric its color. Turmeric is a strong antioxidant, anti-inflammatory, and chemotherapeutic agent. It has been found to inhibit the proliferation and survival of a wide variety of tumor cell types through diverse mechanisms. For example, turmeric inhibits the growth of tumor cells, inhibits replication, and promotes apoptosis by suppressing the activation pathway kappaB. It also inhibits the proliferative effects of BPA on breast cancer cells. Treatment with curcumin, a miR-19 inhibitor (miR-19 is involved in BPA-mediated MCF-7 cell proliferation), leads to suppression of proliferation, growth, and invasion/metastasis of cancer cells. Scientists have actually identified more than 40 biomolecules that are involved in cell death induced by curcumin [15]. Importantly, turmeric targets cancer stem cells (CSCs), a small subset of distinct cells with stem-like character and which are resistant to most chemotherapy drugs as well as radiotherapy. CSCs are the only cancer cells that can metastasize. A study published in the journal Anticancer Research identified the top 25 substances that target cancer stem cells, curcumin being one of them [16]. Its ability to selectively kill cancer cells, including stem cells, but not healthy cells makes it an attractive candidate for both drug development as well as a non-toxic remedy for cancer. Although numerous animal studies and clinical trials have been done, additional studies are needed to learn the full benefits of curcumin.

\section{Licorice root}

Licorice root is one of the most commonly used herbs worldwide, especially in Traditional Chinese Medicine. It has anti-viral, antiinflammatory, hepatoprotective, anti-neurodegenerative, and anticancer properties. Licorice has been found to inhibit various types of cancer. Licorice root stops the proliferation of breast and other cancer cells and modulates the expression of Bcl-2/ Bax apoptotic regulatory factors [17]. It inhibits the production of leukotrienes and prostaglandins, inhibits lymphocytes, and suppresses macrophage phagocytosis. Licorice root provides protection from DNA damage from carcinogens and contains polyphenols which encourage apoptosis in cancer cells. Licorice regulates the production of hormones from the adrenal glands and reduces stress chemicals (chronic stress often triggers the growth of cancer cells). Licorice (and its constituent quercetin discussed previously in phytochemicals) could be an effective antiviral and apoptotic agent for the treatment of virusassociated cancers, including those related to EBV infection, which is linked to the development of many cancers [18]. Glycyrrhizic acid (GA), one of the compounds in licorice root, induces apoptosis of infected cells. GA is currently being used intravenously in Asia for hepatitis infection, and it is plausible that derivatives of GA might be used in the future for treating cancers caused by latent virus infections [19].

\section{Milk thistle}

Milk Thistle (Silybum marianum or silymarin) is an herb with antioxidant and anti-inflammatory properties often used to detoxify the body, especially the liver. Silibinin, a major active flavonoid component of silymarin, consists of a group of flavonoid antioxidants occurring in milk thistle. There have been several studies that have shown milk thistle has anti-cancer effects and appears to have no adverse effects. Milk thistle has been found to have strong in vitro effects against prostate, colon, and lung cancer cells as well as estrogen dependent and estrogen independent breast cancer cells. Memorial Sloan Kettering researchers suggest that milk thistle has anticancer activity against colon and prostate cancer cells [20]. A 2002 study found that milk thistle inhibits cancer growth and promotes apoptosis in rat prostate cancer cells [21]. A 2008 review published in Cancer Letters summarized the multi-targeted chemopreventive and interventive targets and mechanisms of milk thistle in various cancer models, both in vitro and in vivo [22]. According to the researchers, silymarin seems to exert its anticancer effects by multiple molecular mechanisms that could block all stages of carcinogenesis, initiation, promotion, and progression. The researchers further suggest that in particular, anti-invasive and anti-metastatic effects of silymarin authenticate its possible usefulness as preventive and therapeutic agent in the treatment of more advanced and aggressive forms of cancer.

\section{Parsley}

Parsley, a powerful chelating herb, is recognized for its ability to draw out toxic heavy metals and other carcinogens and expel them from the body. It is also an incredible anti-cancer food, rich in flavonoids like myricetin and apigenin that research shows help inhibit cancer cells and tumors and reduce inflammation. The phytochemicals in parsley can slow the speed of cell division, leaving time for the cell to correct DNA mistakes and activate apoptosis and inhibit the migration of cancer cells. Considerable research shows that the flavonoid apigenin, found in celery, artichokes, and parsley may well be a key agent for killing breast, pancreatic, and numerous other cancer cell lines [23]. For example, 2015 research found apigenin to inhibit the growth of breast cancer cells, including HER2-positive ones [24]. 2006 research published in Molecular Cancer found that apigenin inhibits growth of pancreatic cancer cells through suppression of cyclin B-associated cdc2 activity and G2/M cell cycle arrest [25].

\section{Rosemary}

Rosemary helps protect against breast and other cancers and is a powerful activator of detoxification enzymes. Carnosol, a principal component of rosemary, has oncogenic effects including increasing the production of enzymes that detoxify carcinogens, inducing apoptosis, and reducing inflammation. The rosmarinic acid in rosemary deactivates protein 1(AP1), which has been found to be one of the major promotors of colon cancer. A 2015 Spanish study published in Food and Chemical Toxicology found that rosemary leaf extract has antiproliferative activity in human colon cancer cells [26]. A 2016 joint study by Spanish and Swedish researchers published in the Journal of Proteome Research also found rosemary extract to have antiproliferative properties against human colon cancer cells [27]. Research published in 2015 in the Journal PLoS One found that rosemary had an anti-proliferative 
effect on human melanoma cells. Those researchers found that rosemary drastically reduced melanoma cell growth and metabolic activity [28]. A 2016 study reported in Biomedicine \& Pharmacotherapy found rosemary to have considerable antitumor and chemopreventive properties in lung cancer [29].

\section{Oregano}

Oregano possesses anti-bacterial as well as anti-inflammatory properties and promotes cancer cell death, making it a powerful anti-cancer herb. Research shows that the carvacrol in oregano induces apoptosis in prostate cancer cells. While more studies need to be done, researchers at Long Island University concluded that carvacrol has huge potential for use as an anti-cancer agent and possible treatment for prostate cancer [30].

\section{Thyme}

Thyme possesses terpenoids which are recognized for their cancer preventive properties. Rosmarinic and ursolic acids are major terpenoids in thyme that possess anti-cancer properties. Several other anticancer agents in thyme have been identified. These include linalool, borneol, geraniol, sabinene hydrate, thymol, and carvacrol [31]. Studies have shown that thyme is cytotoxic against MCF-7 breast cancer cells, PC-3 prostate cancer cells, lung carcinoma, head and neck squamous cell carcinoma, melanoma, and A549 lung cancer cells [32].

\section{Conclusion}

During the last century, scientific knowledge about anticancer phytochemicals and herbs has remarkably progressed. Despite numerous reports of their effects on cancer, functional use for the prevention and treatment of cancer has been largely limited to self-care. Like most cancer drugs, the main mechanisms of action of plants include inducing apoptosis and inhibiting proliferation, growth, and migration. But unlike conventional cancer drug therapies, phytotherapies are cytotoxic to cancer cells and relatively non-toxic to normal cells. Moreover, use of phytomedicines may be an option for the prevention and treatment of cancer both with and without conventional drugs. In the event that conventional cancer drugs are not accepted due to their side effects or low effectiveness, phytomedicines offer an effective and viable alternative as they are considerably safe and relatively affordable. It is worth recommending application of phytomedicines as alternative cancer therapies for patients who do not benefit or face side effects from conventional drugs or alongside conventional treatments to improve efficacy.

\section{References}

1. David J Newman, Gordon M Cragg. Natural Products as Sources of New Drugs from 1981 to 2014. J Natural Products. 2016;79:629-61.

2. Jin Zhou, Li Fang, Jiaxu Liao, et al. Investigation of the anti-cancer effect of quercetin on HepG2 cells in vivo. PLoS One. 2017;12:e0172838.

3. Lee M, Son M, Ryu E, et al. Quercetin-induced apoptosis prevents EBV infection. Oncotarget. 2015;6:12603-24.

4. Minjung Lee, Myoungki Son, Eunhyun Ryu, et al. Quercetin-induced apoptosis prevents EBV infection. Oncotarget. 2015;6:12603-24.
5. Jang M, Cai L, Udeani GO, et al. Cancer chemopreventive activity of resveratrol, a natural product derived from grapes. Science. 1997;10:275:218-20.

6. Narayanan BA, Narayanan NK, Re GG, et al. Differential expression of genes induced by resveratrol in $\mathrm{LNCaP}$ cells: P53-mediated molecular targets. Int $\mathrm{J}$ Cancer. 2003;104:204-12.

7. Gong WH, Zhao N, Zhang ZM, et al. The inhibitory effect of resveratrol on COX-2 expression in human colorectal cancer: a promising therapeutic strategy. Eur Rev Med Pharmacol Sci. 2017;21:1136-43.

8. Julieta Saluzzo, Kelly M Hallman, Katie Aleck, et al. The regulation of tumor suppressor protein, p53, and estrogen receptor $(\mathrm{ER} \alpha)$ by resveratrol in breast cancer cells. Genes Cancer. 2016;7:414-25.

9. Arnaud Besson, Steven F Dowdy, James M Roberts, et al. CDK Inhibitors: Cell Cycle Regulators and Beyond. Developmental Cell. 2008;14:159-169.

10. Suthat Chottanapund, Van Duursen, PanidaNavasumrit, et al. Anti-aromatase effect of resveratrol and melatonin on hormonal positive breast cancer cells co-cultured with breast adipose fibroblasts. Toxicology in Vitro. 2014;28:1215-1221.

11. Grossarth-Maticek R, Kiene H, Baumgartner SM, et al. Use of Iscador, an extract of European mistletoe (Viscum album), in cancer treatment: prospective nonrandomized and randomized matched-pair studies nested within a cohort study. Altern Ther Health Med. 2001;7:57-66.

12. Giovannucci E. Tomatoes, tomato-based products, lycopene, and cancer: review of the epidemiologic literature. J Natl Cancer Inst. 1999;91:317-31.

13. http://www.aicr.org/press/press-releases/2016/tomatoesother-foods-containing-lycopene-may-protect-againstprostate-cancer-study-finds.html.

14. Walfisch S, Walfisch Y, Kirilov E, et al. Tomato lycopene extract supplementation decreases insulin-like growth factor-I levels in colon cancer patients. Eur J Cancer Prev. 2007;16:298-303.

15. Carini F, David S, Tomasello G, et al. Colorectal cancer: an update on the effects of lycopene on tumor progression and cell proliferation. J Biol Regul Homeost Agents. 2017;31:769-74.

16. Clark LC, Combs GF Jr, Turnbull BW, et al. Effects of selenium supplementation for cancer prevention in patients with carcinoma of the skin. A randomized controlled trial. Nutritional Prevention of Cancer Study Group. JAMA. 1996;276:1957-63.

17. Jayaraj Ravindran, Sahdeo Prasad, Bharat B Aggarwal, et al. Curcumin and Cancer Cells: How Many Ways Can Curry Kill Tumor Cells Selectively? AAPS J. 2009;11:495510.

18. http://www.greenmedinfo.com/article/natural-productstarget-cancer-stem-cells

19. Jo EH, Hong HD, Ahn NC, et al. Modulations of the Bcl-2/ 
Bax family were involved in the chemopreventive effects of licorice root (Glycyrrhiza uralensis Fisch) in MCF-7 human breast cancer cell. J Agric Food Chem. 2004;52:1715-9.

20. h t t p s : / / w w w s c i e n c e d a i 1 y. c o m / releases/2017/02/170213131346.htm

21. Jeffrey I Cohen. Licking latency with licorice. J Clin Invest. 2005;115:591-3.

22. https://www.mskcc.org/cancer-care/integrative-medicine/ herbs/milk-thistle

23. Alpana Tyagi, Neehar Bhatia, Mark S. Antiproliferative and apoptotic effects of silibinin in rat prostate cancer cells. The Prostrate. 2002;53:211-17.

24. Kumaraguruparan Ramasamy, Rajesh Agarwa. Multitargeted therapy of cancer by silymarin. Cancer Lett. 2008;269:352-62.

25. Sanjeev Shukla, Sanjay Gupta. Apigenin: A Promising Molecule for Cancer Prevention. Pharm Res. 2010;27:962-78.

26. Scherbakov AM, Andreeva OE. Apigenin Inhibits Growth of Breast Cancer Cells: The Role of ER $\alpha$ and HER2/neu. Acta Naturae. 2015;7:133-9.

27. Michael B Ujiki, Xian-Zhong Ding, M Reza Salabat, et al. Apigenin inhibits pancreatic cancer cell proliferation through G2/M cell cycle arrest. Mol Cancer. 2006;5:76.

28. Borrás-Linares I, Pérez-Sánchez A, Lozano-Sánchez J, et al. A bioguided identification of the active compounds that contribute to the antiproliferative/cytotoxic effects of rosemary extract on colon cancer cells. Food Chem Toxicol. 2015;80:215-22.

29. Valdés A, Artemenko KA, Bergquist J, et al. Comprehensive Proteomic Study of the Antiproliferative Activity of a Polyphenol-Enriched Rosemary Extract on Colon Cancer Cells Using Nanoliquid Chromatography-Orbitrap MS/MS. J Proteome Res. 2016;15:1971-85.

30. Cattaneo L, Cicconi R, Mignogna G, et al. AntiProliferative Effect of Rosmarinus officinalis L. Extract on Human Melanoma A375 Cells. PLoS One. 2015 Jul. 15;10(7):e0132439

31. Moore J, Megaly M, MacNeil AJ, et al. Rosemary extract reduces $\mathrm{Akt} / \mathrm{mTOR} / \mathrm{p} 70 \mathrm{~S} 6 \mathrm{~K}$ activation and inhibits proliferation and survival of A549 human lung cancer cells. Biomed Pharmacother. 2016;83:725-32.

32. http://www.liu.edu/Brooklyn/About/News/Campus-PressReleases/2012/April/BK-PR-Apr25-2012.

\section{*Correspondence to:}

Elyn C Jacobs

Former Executive Director, Emerald Heart Cancer Foundation, USA

E-mail: elyn@elynjacobs.com 